\title{
Defective telomere elongation and hematopoiesis from telomerase-mutant aplastic anemia iPSCs
}

\author{
Thomas Winkler, ${ }^{1}$ So Gun Hong, ${ }^{1}$ Jake E. Decker, ${ }^{1,2}$ Mary J. Morgan, ${ }^{1}$ Chuanfeng Wu, ${ }^{1}$ \\ William M. Hughes V, ${ }^{1}$ Yanqin Yang, ${ }^{3}$ Danny Wangsa, ${ }^{4}$ Hesed M. Padilla-Nash, ${ }^{4}$ \\ Thomas Ried, ${ }^{4}$ Neal S. Young, ${ }^{1}$ Cynthia E. Dunbar, ${ }^{1}$ and Rodrigo T. Calado, ${ }^{1,5}$ \\ ${ }^{1}$ Hematology Branch, National Heart Lung and Blood Institute (NHLBI), NIH, Bethesda, Maryland, USA. \\ ${ }^{2}$ Howard Hughes Medical Institute, Chevy Chase, Maryland, USA. 'DNA Sequencing and Genomics Core Facility, \\ NHLBI, and ${ }^{4}$ Section of Cancer Genomics, Genetics Branch, National Cancer Institute, NIH, Bethesda, Maryland, USA. \\ ${ }^{5}$ Department of Internal Medicine, University of São Paulo at Ribeirão Preto Medical School, Ribeirão Preto, São Paulo, Brazil.
}

\begin{abstract}
Critically short telomeres activate p53-mediated apoptosis, resulting in organ failure and leading to malignant transformation. Mutations in genes responsible for telomere maintenance are linked to a number of human diseases. We derived induced pluripotent stem cells (iPSCs) from 4 patients with aplastic anemia or hypocellular bone marrow carrying heterozygous mutations in the telomerase reverse transcriptase (TERT) or the telomerase RNA component (TERC) telomerase genes. Both mutant and control iPSCs upregulated $T E R T$ and TERC expression compared with parental fibroblasts, but mutant iPSCs elongated telomeres at a lower rate compared with healthy iPSCs, and the deficit correlated with the mutations' impact on telomerase activity. There was no evidence for alternative lengthening of telomere (ALT) pathway activation. Elongation varied among iPSC clones derived from the same patient and among clones from siblings harboring identical mutations. Clonal heterogeneity was linked to genetic and environmental factors, but was not influenced by residual expression of reprogramming transgenes. Hypoxia increased telomere extension in both mutant and normal iPSCs. Additionally, telomerase-mutant iPSCs showed defective hematopoietic differentiation in vitro, mirroring the clinical phenotype observed in patients and demonstrating that human telomere diseases can be modeled utilizing iPSCs. Our data support the necessity of studying multiple clones when using iPSCs to model disease.
\end{abstract}

\section{Introduction}

Telomeres are nucleoprotein structures at the end of linear chromosomes consisting of repetitive, non-protein coding DNA sequences that are coated by the protein complex shelterin (1). Telomeres protect the end of the chromosomes from DNA damage and prevent the activation of DNA-damage signaling pathways and nonhomologous end joining. In humans, telomeric DNA is composed of TTAGGG tandem repeats. Telomerase consists of a reverse transcriptase enzyme (TERT), an RNA template (TERC), and stabilizing proteins including dyskerin (encoded by DKC1) and TCAB1 (2). The enzymatic complex adds nucleotide hexamers to the $3^{\prime}$ end of telomeres in highly proliferative cells.

Disturbed telomere homeostasis is etiologic in several severe human diseases (3). In X-linked dyskeratosis congenita, critically short telomeres are due to mutations in the X-linked DKC1 gene. Although clinically characterized by a triad of mucocutaneous findings (nail dystrophy, leukoplasia, and reticular skin hypopigmentation), dyskeratosis congenita is a pleiotropic, multi-organ disease, and the majority of the patients succumb to bone marrow failure (80\%), pulmonary fibrosis, or liver disease (4). The clinical diagnosis of dyskeratosis congenita requires the presence of at least 2 features of the mucocutaneous triad (5).

Authorship note: Thomas Winkler, So Gun Hong, and Jake E. Decker contributed equally to this work.

Conflict of interest: The authors have declared that no conflict of interest exists. Citation for this article: J Clin Invest. 2013;123(5):1952-1963. doi:10.1172/JCI67146.
We and others have demonstrated that heterozygous mutations in TERT or TERC result in very short telomeres of leukocytes, translating into phenotypes that are milder and clinically distinct from those observed in dyskeratosis congenita; heterozygous TERC or TERT mutations often affect a single organ, the clinical presentation is later in life, and patients usually lack the physical abnormalities typical of dyskeratosis congenita. Telomerase mutations are genetic risk factors for apparently acquired aplastic anemia (6), idiopathic pulmonary fibrosis $(7,8)$, liver disorders (9-11), and acute myeloid leukemia $(12,13)$. The clinical phenotype of individuals within families harboring a given mutation is variable, extending from asymptomatic carriers through mild laboratory findings to severe aplastic anemia (clinically indistinguishable from acquired aplastic anemia and without the accompanying physical examination features of dyskeratosis congenita). Patients and other family members with mutations may have subtle or severe organ involvement beyond the marrow, especially pulmonary fibrosis or hepatic cirrhosis. In contrast to the high genetic penetrance observed in X-linked dyskeratosis congenita, other genetic, epigenetic, and environmental factors appear to modulate disease phenotype in these telomeropathies (3). Dyskeratosis congenita represents the most severe phenotype, but probably represents a small portion of the expanding spectrum of illnesses caused by telomere dysfunction.

In the adult organism, telomerase expression is tightly regulated and mainly restricted to stem and progenitor cells responsible for replenishing actively proliferating tissues such as the bone marrow. Investigating the molecular mechanisms and environ- 


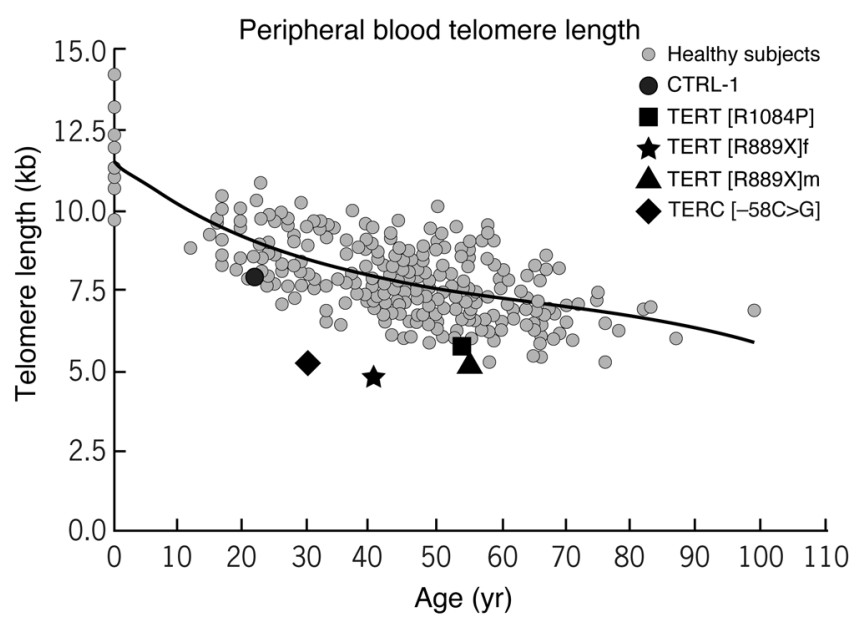

Figure 1

Blood leukocyte telomere length $(\mathrm{kb})$ as a function of age. Individuals with TERC and TERT mutations have very short telomeres in peripheral blood leukocytes. The line designates the 50th percentile of telomere length for 298 healthy blood donors (25). The peripheral blood leukocyte telomere lengths were measured by $\mathrm{qPCR}$ and T/S ratios transformed to kilobases based on a linear correlation between qPCR and Southern blot results $\left(r^{2}=0.86\right)$.

mental modulators of telomerase expression that translate into disease phenotype is difficult because the primary affected cells, for instance, bone marrow stem and progenitor cells in aplastic anemia, are markedly depleted.

The recent technology pioneered by Takahashi and Yamanaka (14) of reprogramming somatic cells into a pluripotent, ES celllike phenotype offers a method for modeling telomere dynamics in the telomeropathies and for investigating pathways resulting in tissue dysfunction. High expression of telomerase in human ES cells ensures maintenance of telomere length and helps define their pluripotent phenotype (15). Telomerase is also upregulated during iPSC generation $(16,17)$ and is likely responsible for telomere elongation and maintenance in these cells $(18,19)$. Therefore, reprogramming technology followed by differentiation could be a valuable tool for studying telomere dynamics in a mutation- and patient-specific manner. Based on their pluripotent phenotype and ability to differentiate into virtually any tissue type, induced pluripotent stem cells (iPSCs) may enable the study of potential environmental or epigenetic factors responsible for the variable penetrance of telomere diseases.

Recently, 2 studies investigating telomere dynamics in iPSCs derived from an $\mathrm{X}$-linked dyskeratosis congenita patient with a loss-of-function mutation in the DKC1 gene came to contradictory conclusions. Albeit at very low efficiency, both studies successfully derived iPSCs from patients' fibroblasts. Despite the presumable loss of function of DKC1, Agarwal et al. reported telomere elongation in iPSCs (20) and TERT and TERC upregulation, whereas Batista et al. observed reduced telomerase activity and progressive telomere erosion, associated with loss of self-renewal potential and early senescence (21). Batista et al. also found in iPSCs derived from dyskeratosis congenita patients with TERT heterozygous mutations that telomerase activity was reduced and telomere elongation during reprogramming was impaired. However, neither study explored the telomere dynamics in iPSCs from patients with more common and milder telomere diseases than dyskeratosis congenita.
In an effort to develop an in vitro iPSC model for patients with aplastic anemia who do not display the typical dyskeratosis congenita phenotype but have very short telomeres due to heterozygous loss-of-function mutations in telomerase complex genes, we derived iPSCs from patients with hematologic abnormalities harboring mutations in the TERT or TERC genes. Given the conflicting results in prior studies as to whether iPSCs accurately model telomere dynamics in dyskeratosis congenita, we generated several iPSC clones from each of multiple patients with mutations in TERT or TERC and studied their telomere dynamics. We measured telomerase gene expression and telomerase enzymatic activity at multiple time points during the reprogramming process and after prolonged passaging. Additionally, we investigated the impact of environmental factors on telomere maintenance, specifically hypoxia, which had been shown to increase reprogramming efficiency and to reduce differentiation in ES cells $(22,23)$. We also investigated whether telomere elongation during reprogramming exclusively depends on telomerase or whether recombinationmediated alternative lengthening of telomere (ALT) contributes to telomere length maintenance in iPSCs.

\section{Results}

Patient characteristics and iPSC derivation. Four adult patients with aplastic anemia or markedly reduced bone marrow cellularity, ageadjusted markedly short telomeres, and known mutations within the telomerase complex genes were studied (Figure 1 and Table 1). Three patients had TERT mutations, including 2 siblings with a reverse transcriptase domain mutation abolishing telomerase function (TERT[R889X]f and TERT[R889X]m) and a patient with C-terminal extension domain mutation of TERT (TERT[R1084P]), reducing activity by $70 \%(24)$. One patient had a heterozygous TERC promoter mutation in the CCAAT box (TERC[-58C>G]) (25), resulting in significantly decreased gene expression. Control iPSCs were generated from a normal volunteer with no family history or manifestations of telomere diseases (control 1) and commercially available newborn foreskin fibroblast cells (control 2).

iPSCs were generated by overexpression of the transcription factors POU5F1 (OCT4), SOX2, KLF4, and MYC individually from retroviral vectors (26) (retroviral OSKM) or from a lentiviralbased polycistronic vector (27) (polycistronic OSKM, STEMCCA) in skin fibroblasts. Multiple iPSC clones could be obtained from each patient or control. All derived iPSCs had typical ES cell morphology, stained positive for alkaline phosphatase and the pluripotency markers OCT4, SSEA4, NANOG, Tra1-60, and Tra1-81 by immunofluorescence (Supplemental Figure 1A; supplemental material available online with this article; doi:10.1172/ JCI67146DS1), and formed teratomas in NSG mice and formed teratomas in NSG mice (Supplemental Figure 1B). Additionally, microarray mRNA expression studies on control (control 2) and all telomerase mutant iPSCs confirmed an ES cell-like mRNA expression profile (Supplemental Figure 1C; GEO GSE42869). Supplemental Table 1 summarizes all iPSC characterization studies performed. Sequence analysis documented that all iPSCs retained the original telomerase mutation present in the parental fibroblasts (Supplemental Figure 2). Spectral karyotyping (SKY) was performed on selected iPSCs (28). The composite karyotypes are presented in Supplemental Table 4.

Impaired telomere elongation in mutant iPSCs. Using quantitative PCR (qPCR) (29), telomere length was measured for at least 20 passages in all clones. We found that telomeres elongated in all 
Table 1

Patient characteristics and iPSC selected for further analysis

\begin{tabular}{|c|c|c|c|c|c|}
\hline Patient (mutation) & $\begin{array}{l}\text { Effect of mutation on } \\
\text { enzyme activity }\end{array}$ & Clinical manifestation & Family history & iPSC clone & Reprogramming method \\
\hline $\begin{array}{l}53 \text { years old, male } \\
\text { TERT[R1084P] }\end{array}$ & $\begin{array}{l}\text { Enzyme activity } \\
\text { reduced by } 70 \%\end{array}$ & $\begin{array}{l}\text { Hypocellular bone } \\
\text { marrow }(<5 \%) \text { with } \\
\text { normal PBC counts }\end{array}$ & $\begin{array}{l}\text { Pulmonary } \\
\text { fibrosis }\end{array}$ & $\begin{array}{l}c 5.1 \\
c 7 \\
c 13\end{array}$ & $\begin{array}{l}\text { Retroviral, OSKM } \\
\text { Retroviral, OSKM } \\
\text { Retroviral, OSKM }\end{array}$ \\
\hline $\begin{array}{c}45 \text { years old, female } \\
\text { TERTIR889X]f }\end{array}$ & $\begin{array}{l}\text { Telomerase function } \\
\text { abolished }\end{array}$ & $\begin{array}{c}\text { Progressive aplastic } \\
\text { anemia }\end{array}$ & $\begin{array}{l}\text { Pulmonary } \\
\text { fibrosis }\end{array}$ & $\begin{array}{l}c 2 \\
c 4.1 \\
\text { c20 }\end{array}$ & $\begin{array}{c}\text { Retroviral, OSKM } \\
\text { Retroviral, OSKM } \\
\text { Polycistronic, OSKM }\end{array}$ \\
\hline $\begin{array}{l}55 \text { years old, male, } \\
\text { TERT [R889X]m } \\
\text { (brother of } 45 \text { years } \\
\text { TERT[R889X]f) }\end{array}$ & $\begin{array}{l}\text { Telomerase function } \\
\text { abolished }\end{array}$ & $\begin{array}{l}\text { Hypocellular bone } \\
\text { marrow }(<5 \%) \\
\text { with normal } \\
\text { PBC counts }\end{array}$ & $\begin{array}{l}\text { Pulmonary } \\
\text { fibrosis }\end{array}$ & c20 & Polycistronic OSKM \\
\hline $\begin{array}{c}30 \text { years old, female } \\
\text { TERC }[-58 C>G]\end{array}$ & $\begin{array}{c}\text { Allelic reduced } \\
\text { expression of TERC }\end{array}$ & Aplastic anemia & Aplastic anemia & $\begin{array}{l}\mathrm{c} 13 \\
\mathrm{c} 14 \\
\mathrm{c} 18\end{array}$ & $\begin{array}{l}\text { Polycistronic 0SKM } \\
\text { Polycistronic OSKM } \\
\text { Polycistronic OSKM }\end{array}$ \\
\hline $\begin{array}{l}22 \text { years old, male } \\
\text { [CTRL-1] }\end{array}$ & $\mathrm{N} / \mathrm{A}$ & Clinically healthy & Empty for telomere diseases & $\begin{array}{l}c 2 \\
c 3\end{array}$ & $\begin{array}{l}\text { Retroviral, OSKM } \\
\text { Retroviral, OSKM }\end{array}$ \\
\hline $\begin{array}{l}\text { BJ-1, newborn foreskin } \\
\text { (CRL-2522, ATCC, } \\
\text { [CTRL-2]) }\end{array}$ & $\mathrm{N} / \mathrm{A}$ & $\mathrm{N} / \mathrm{A}$ & $\mathrm{N} / \mathrm{A}$ & $\begin{array}{l}\mathrm{c} 26 \\
\mathrm{c} 1 \\
\mathrm{c} 4\end{array}$ & $\begin{array}{c}\text { Polycistronic OSKM } \\
\text { Retroviral, OSKM } \\
\text { Retroviral, OSKM }\end{array}$ \\
\hline
\end{tabular}

OSKM, OCT4, SOX2, KLF4, MYC; polycistronic, OSKM expressed from the single polycistronic lentiviral construct STEMCCA.

iPSCs compared with their parental fibroblasts (Figure 2); results were validated by standard Southern hybridization (Figure 2, $\mathrm{H}$ and $\mathrm{I}$ ). However, the telomere elongation rate was substantially lower in the TERT- and TERC-mutant iPSCs as compared with iPSCs derived from healthy controls. Telomere extension of heterozygous telomerase-mutant iPSCs was approximately half of that observed in healthy control iPSCs (Figure 2G). However, the telomere elongation pattern was substantially heterogeneous among iPSC clones from different patients and even among independent clones derived from the same patient (Figure 2). All TERTand TERC-mutant iPSC clones, except for clone TERT[R889X]f c4.1, elongated telomeres at various rates for the first 15 passages and then reached a plateau.

TERT [R889X] mutation reduces TERT RNA expression. We investigated how reprogramming affected TERT and TERC gene expression. All iPSC clones (from mutant patients and controls) had marked and comparable increases in both TERT and TERC mRNA expression levels in comparison with parental fibroblasts (Figure 3, $\mathrm{A}$ and $\mathrm{B}$ ). TERC expression was lower in TERT-mutant iPSC clones in comparison with healthy controls (Figure 3C). Although TERT mRNA expression was comparable between controls and iPSC clones with TERT[R1084P] or TERC[-58C>G] mutations, TERT expression was significantly lower in iPSCs derived from both patients carrying the truncation mutation in TERT [R889X] $(P<0.05$; Figure 3C). We also observed variability in mRNA expression of both TERT and TERC between clones derived from the same donor and at successive passages even in an individual clone (Figure 3, A and B).

Transgene excision does not modulate the expression of TERT and TERC. It is possible that permanent or transient reactivation of reprogramming transgenes contributes to the telomere elongation and the observed heterogeneity, especially after the recent observation that the $\mathrm{Wnt} / \beta$-catenin pathway activates the TERT gene (30) via interaction with KLF4 in a murine ESC model and in human cancer cell lines. However, in multiple iPSC clones derived from controls and telomerase-mutant patients, analysis of the $\beta$-catenin canonical pathway via gene expression profiling did not correlate with the rate of telomere elongation (GEO GSE42869). Furthermore, cre-excision of the transgenes expressed from the polycistronic lentiviral vector (Supplemental Figure 3) did not result in reduced expression of TERT and TERC (Figure 3D), indicating that potential residual MYC or KLF4 expression was not responsible for telomerase activation or the heterogeneity in telomere elongation observed among iPSC clones. Indeed, we observed a similar variability in the expression of both genes among different clones and passages of the transgene-free mutant iPSCs (Supplemental Figure 4, A and B).

Telomerase activity and ALT. We determined telomerase functional activity in iPSCs. There was a significant increase in telomerase activity in all iPSCs grouped by mutation type in comparison with parental fibroblasts, comparable to the level seen in ESCs, but also displaying some variation in activity at successive passages as well as in different clones from the same individual (Figure 4A and Supplemental Figure 5). However, telomerase activity did not directly correlate with TERT or TERC mRNA expression.

The decreased telomere elongation rates and reduced telomerase function in heterozygous telomerase-mutant iPSCs, both corresponding to the degree of telomerase deficiency predicted by the gene lesion, suggested that telomere elongation in iPSCs is directly dependent on a functional telomerase complex. Telomerase-independent ALT mechanisms have been reported in some cancer cells (reviewed in ref. 31). The accumulation of C-rich partially single-stranded circles of telomeric DNA (C-circles) is a sensitive and quantifiable marker for ALT (32). In order to detect any contribution of the ALT pathway to telomere extension during reprogramming, we applied the $\mathrm{C}$-circle assay, but found no significant accumulation of $\mathrm{C}$ circles in healthy or mutant iPSCs (Figure 4B). Additionally, increased telomere length heterogeneity is a hallmark of active ALT mechanisms. We did not observe increased telomere length heterogeneity in healthy control or 
A
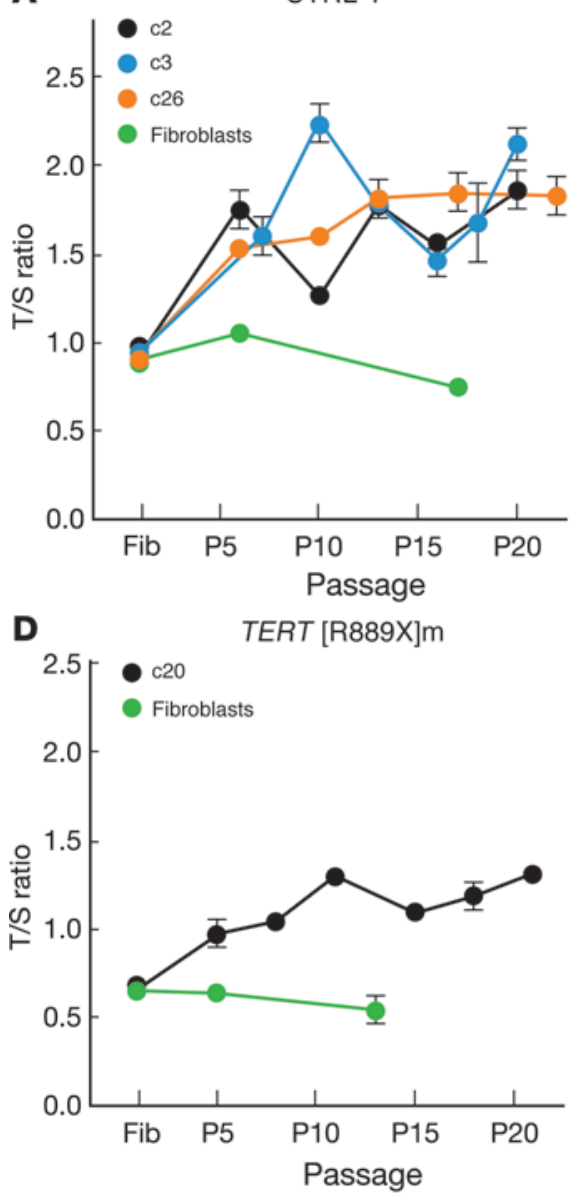

B

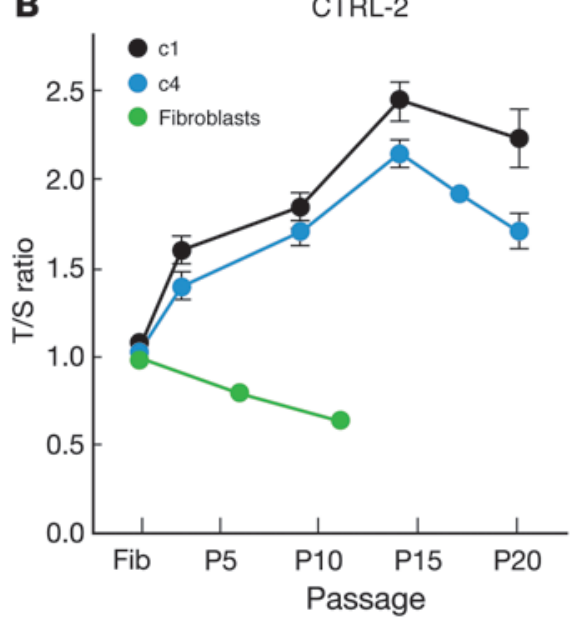

E

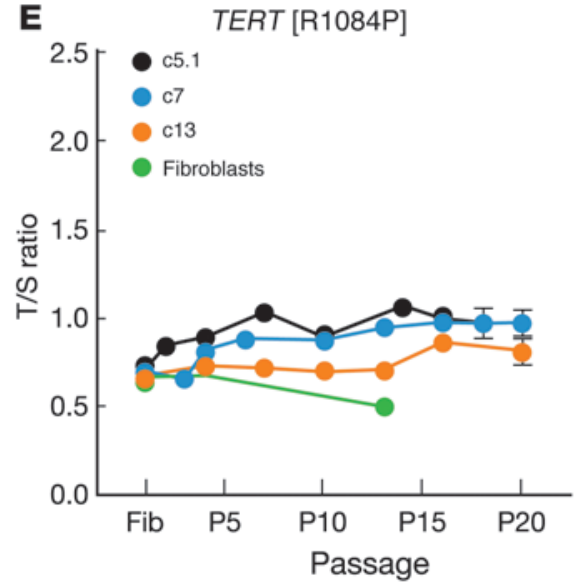

C

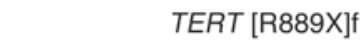

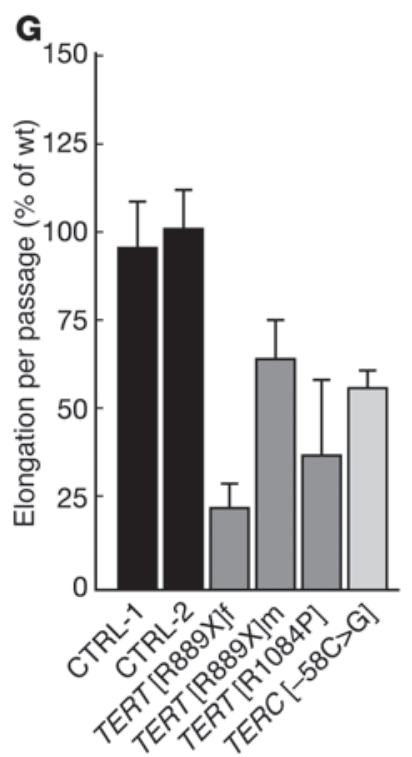
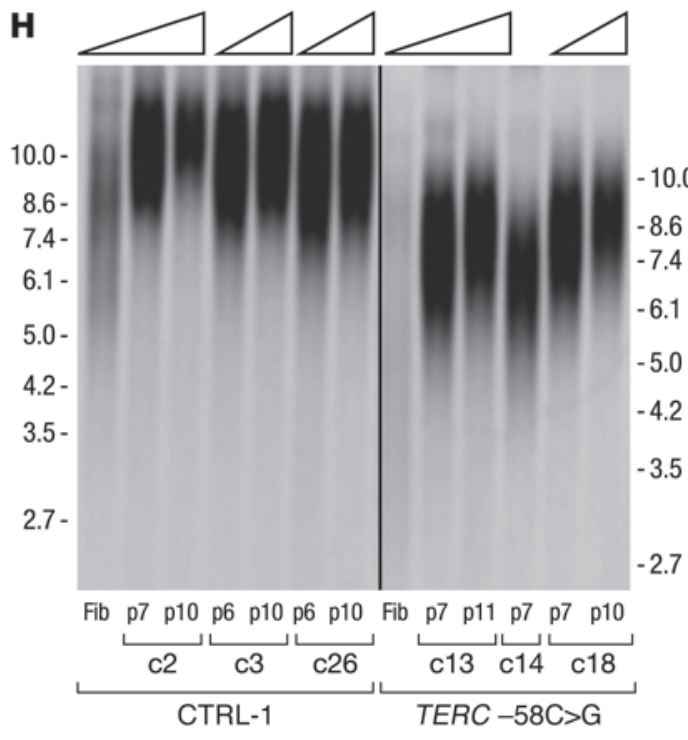

I
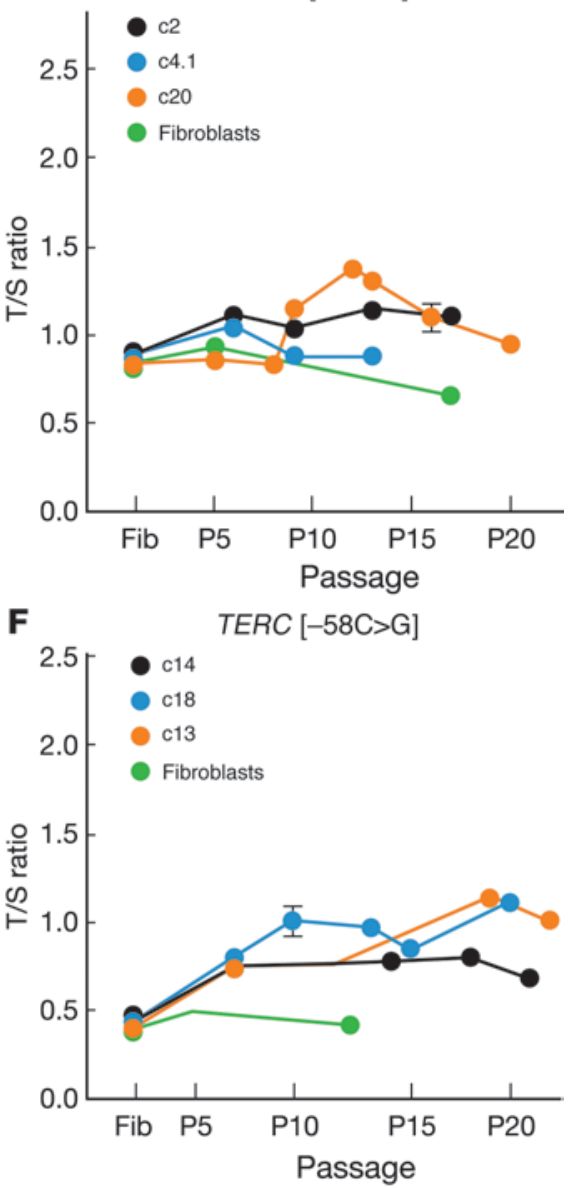

$\mathbf{F}$
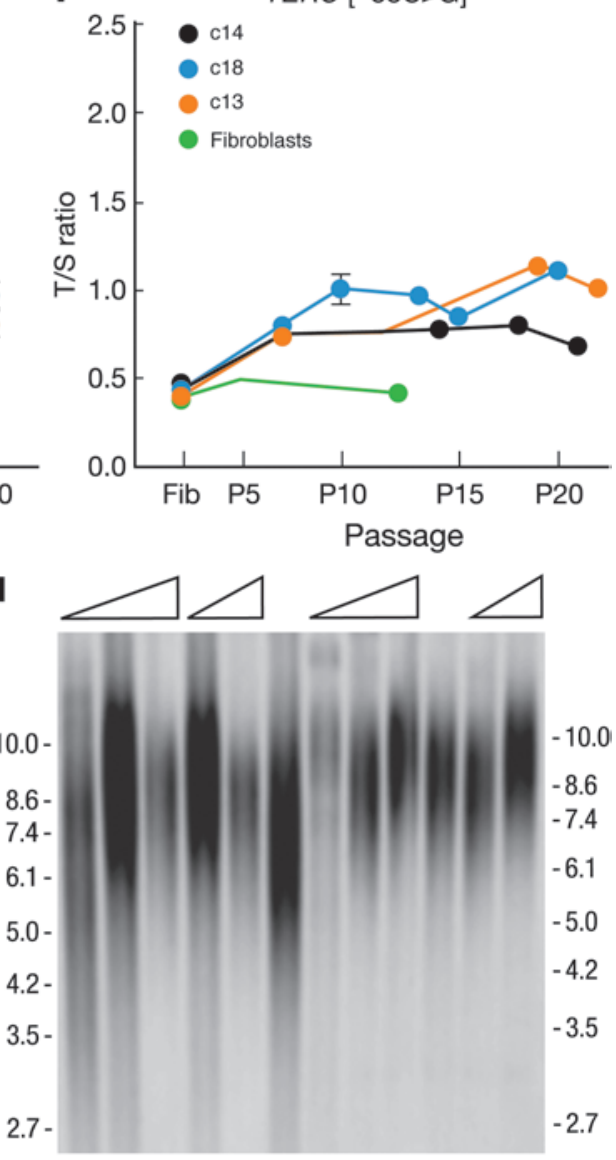

$\begin{array}{lllllllllllll}\text { Fib } & \text { p7 } & \text { p10 } & \text { p6 } & \text { p10 } & \text { p6 } & \text { p10 } & \text { Fib } & \text { p7 } & \text { p11 } & \text { p7 } & \text { p7 } & \text { p10 }\end{array}$

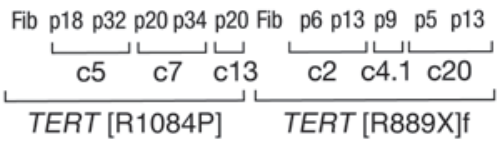

\section{Figure 2}

Telomere lengths in iPSCs derived from controls and from patients with telomerase complex mutations followed over time. (A-F) Each panel shows telomere lengths (represented as T/S ratios) of cells from an individual patient or control. Each line shows values in relation to passage number from independent iPSC clones. In each panel, parental somatic cell lengths are shown in green. To quantify changes in telomere length over time, slopes were generated by linear regression analysis of the T/S ratios during the first 10 passages. (G) Combined elongation slopes from all clones derived from a particular patient or healthy control. Note: TERT[R889X]f and TERTTR889X]m clones are derived from a brother/ sister pair. The suffixes indicate either female $(\mathrm{f})$ or male $(\mathrm{m})$. Southern blot analysis of telomere length in early passages from control, TERC $(\mathbf{H})$, and TERT (I) mutated iPSC confirming qPCR results. Fib, fibroblasts. 

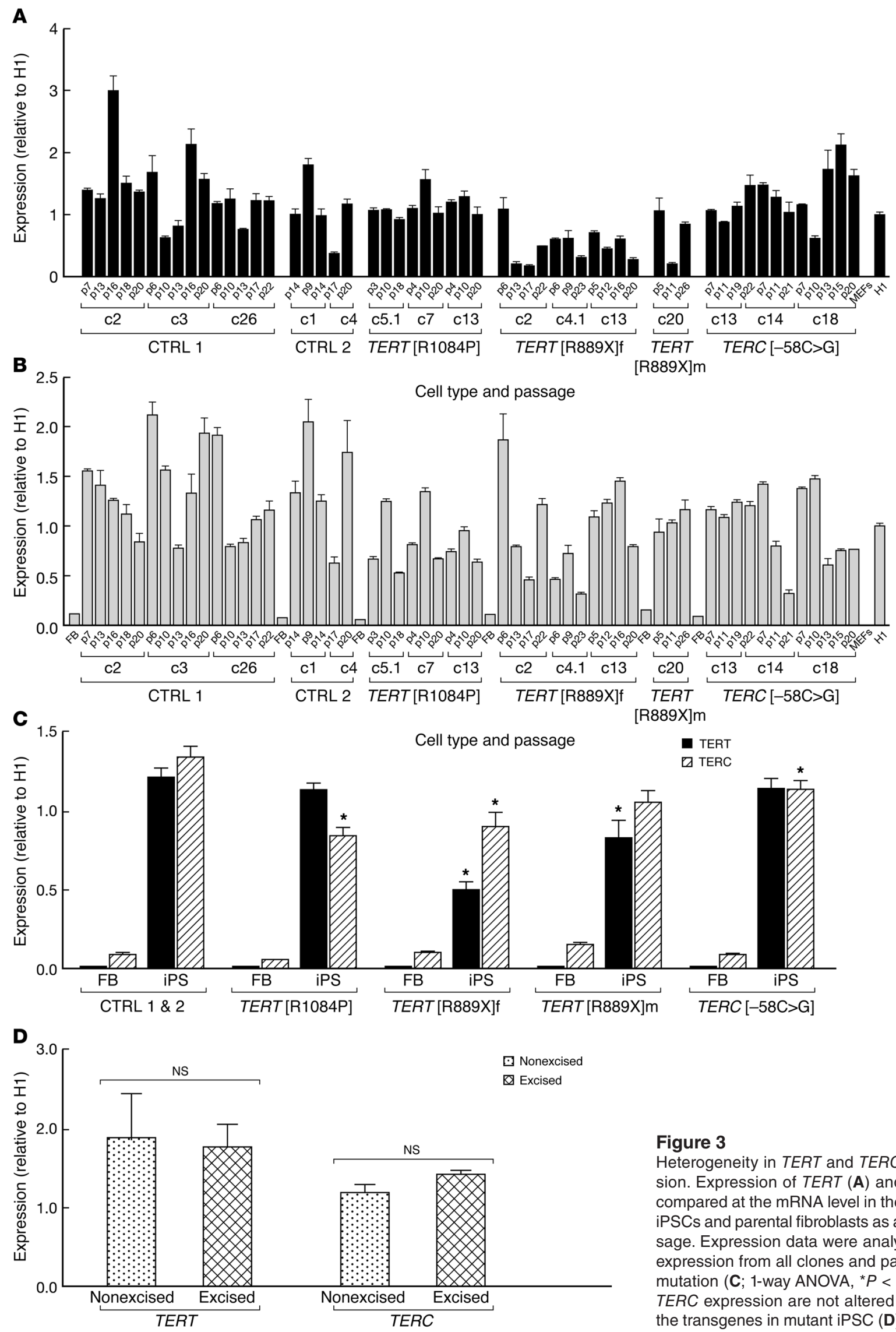

Figure 3

Heterogeneity in TERT and TERC mRNA expression. Expression of TERT (A) and TERC (B) was compared at the mRNA level in the patient-derived iPSCs and parental fibroblasts as a function of passage. Expression data were analyzed as average expression from all clones and passages for each mutation (C; 1-way ANOVA, $\left.{ }^{\star} P<0.05\right)$. TERT and $T E R C$ expression are not altered after excision of the transgenes in mutant iPSC (D). FB, fibroblasts. 
A

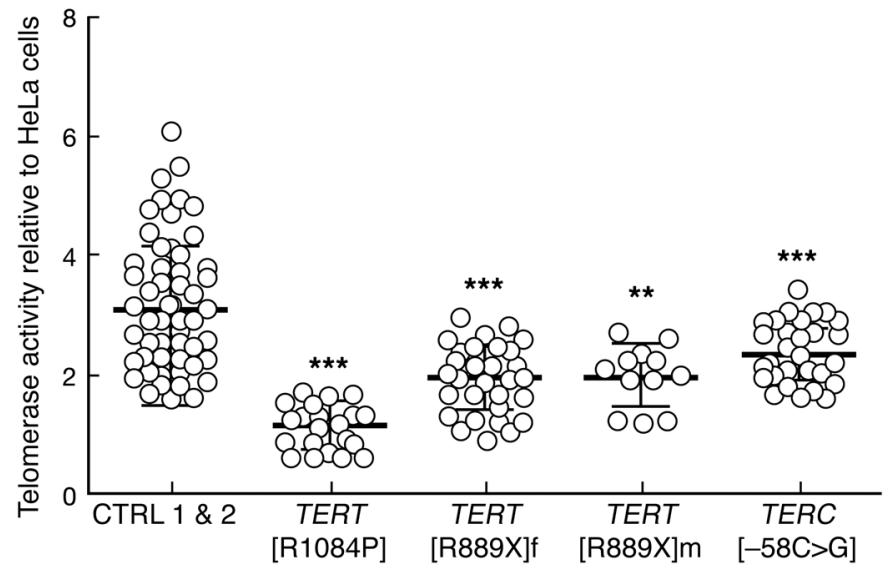

B

TERT [R889X]f c4.1,p7

TERT [R889X]f c2,p7

TERT [R889X] fibroblasts

CTRL-1 fibroblasts

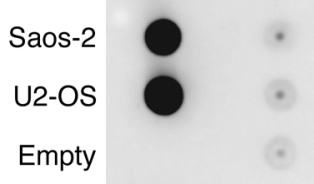

$\mathrm{H}_{2} \mathrm{O}$
CTRL-2 c1,p32

H1, p56

TERC $[-58 \mathrm{C}>\mathrm{G}] \mathrm{c} 13, \mathrm{p} 38,21 \% \mathrm{O}_{2}$

TERC $[-58 \mathrm{C}>\mathrm{G}] \mathrm{c} 13, \mathrm{p} 38,5 \% \mathrm{O}_{2}$

TERT [R1084P] c5.1, p66, 21\% $\mathrm{O}_{2}$ TERT [R1084P] c5.1, p66, 5\% $\mathrm{O}_{2}$

TERT [R889X]m c20, p23

TERT [R889X]f c20, p6

\section{Figure 4}

Telomerase activity and ALT. (A) Telomerase activity was measured and normalized to the activity of HeLa cells. Reactions were performed in triplicate. Data were analyzed as average activity of passages of all clones from a particular mutation or control. Each circle represents 1 data point of measured telomerase activity. (B) Dot blot of C-circle assay performed on genomic DNA from TERT and TERC mutant as well as control IPSCs cultured either in $5 \%$ or $21 \%$ oxygen. Genomic DNA from the ALT-positive cell lines Saos2 and U2-OS served as positive controls. ${ }^{* \star} P<0.01$; ${ }^{* * *} P<0.001$, 1-way ANOVA.

telomerase-mutant iPSCs (Figure 2, H and I). Taken together, these results indicate that telomerase is the only major mechanism responsible for telomere elongation during human cell reprogramming to a pluripotent state.

Telomere maintenance in iPSCs is influenced by environmental and genetic factors. Pluripotency and reprogramming efficiency may be influenced by conditions that alter the intracellular redox state (23), but the impact of ambient oxygen concentration on telomere dynamics in human iPSCs has not previously been investigated. We cultured iPSCs from healthy subjects and from patients with a telomerase complex mutation in hypoxic $(5 \%)$ or ambient oxygen (21\%). After 4 passages, telomeres were longer in both control and telomerase complex mutant iPSC clones cultured at low oxygen levels, in comparison with the same clones cultured in ambient oxygen $(P=0.001$; Figure 5A). In an independent reverse approach, iPSCs previously kept at $5 \%$ oxygen were either maintained at hypoxic conditions or transferred to $21 \%$ oxygen, and similarly, iPSCs transferred to $21 \%$ oxygen had telomeres much shorter than those maintained at $5 \%$ oxygen (Figure $5 \mathrm{~B}$ ). Furthermore, iPSCs grown in low (5\%) oxygen tension had higher TERT mRNA expression in comparison with TERT expression in iPSCs cultured in $21 \%$ oxygen (Figure 5C). However, TERC expression was not altered in response to different oxygen concentrations (data not shown). These observations indicate that low oxygen tension resulted in higher telomerase expression in human iPSCs and enhanced telomere extension, regardless of telomerase mutation status.

We observed that one clone carrying the TERT truncation mutation R889X (clone 4.1) showed sudden erosion of telomere length beginning at passage 9 , in contrast with 2 other clones derived from the same patient's fibroblasts, used to derive the iPSCs (Figure 5D). This accelerated telomere attrition was accompanied by an obvious increase in spontaneous differentiation that eventually precluded further passaging. SKY revealed an unbalanced translocation between chromosomes $\mathrm{X}$ and $2(46, \mathrm{XX}, \operatorname{der}[\mathrm{X}], \mathrm{t}[\mathrm{X}, 2]$ [p22.3;p23]) at the time of accelerated telomere attrition (p26) (Figure 5, D and E). Telomere attrition was confirmed by Southern blot hybridization (Supplemental Figure 6).

Impaired hematopoietic differentiation in telomerase mutant iPSCs. We differentiated control iPSCs and mutated iPSCs toward hematopoietic progenitors via a multistep differentiation procedure (Figure 6A). iPSCs derived from patients with TERT or TERC mutations and aplastic anemia showed embyroid body (EB) formation comparable to control iPSCs and ESCs (Figure 6B). However, in the telomerase mutant iPSC, the percentage of hematopoietic progenitors $\left(\mathrm{CD} 34^{+} \mathrm{CD}_{45^{+}}\right)$on day 20 after EB formation was reduced (Figure $6 \mathrm{C}$ ) as well as the numbers of hematopoietic CFUs (Figure 6D), indicating an impaired hematopoietic differentiation capacity. Moreover, the differences in CFU numbers reflected the different severities of the patients' clinical hematopoietic phenotypes (Table 1), which was mostly apparent in the brother/sister pair with the TERT[R889X] mutation. The sister, with clinical severe aplastic anemia, had profoundly depressed CFU formation from her iPSCs in comparison with her brother's much more moderate defect in this assay of hematopoietic progenitors.

\section{Discussion}

In the present study, we show that adult somatic cells from patients with bone marrow failure syndromes associated with mutations in genes of the telomerase complex are reprogrammable to pluripotency, but telomerase function and telomere elongation remain defective after reprogramming and hematopoietic differentiation is impaired. Impairment in telomere length maintenance in patient iPSCs may depend on the mutated gene and its pattern of influence on telomerase activity, but telomere elongation may also be influenced by genetic abnormalities acquired during reprogramming, clone heterogeneity due to factors involved in iPSC generation and maintenance, and culture conditions such as oxygen concentration. 

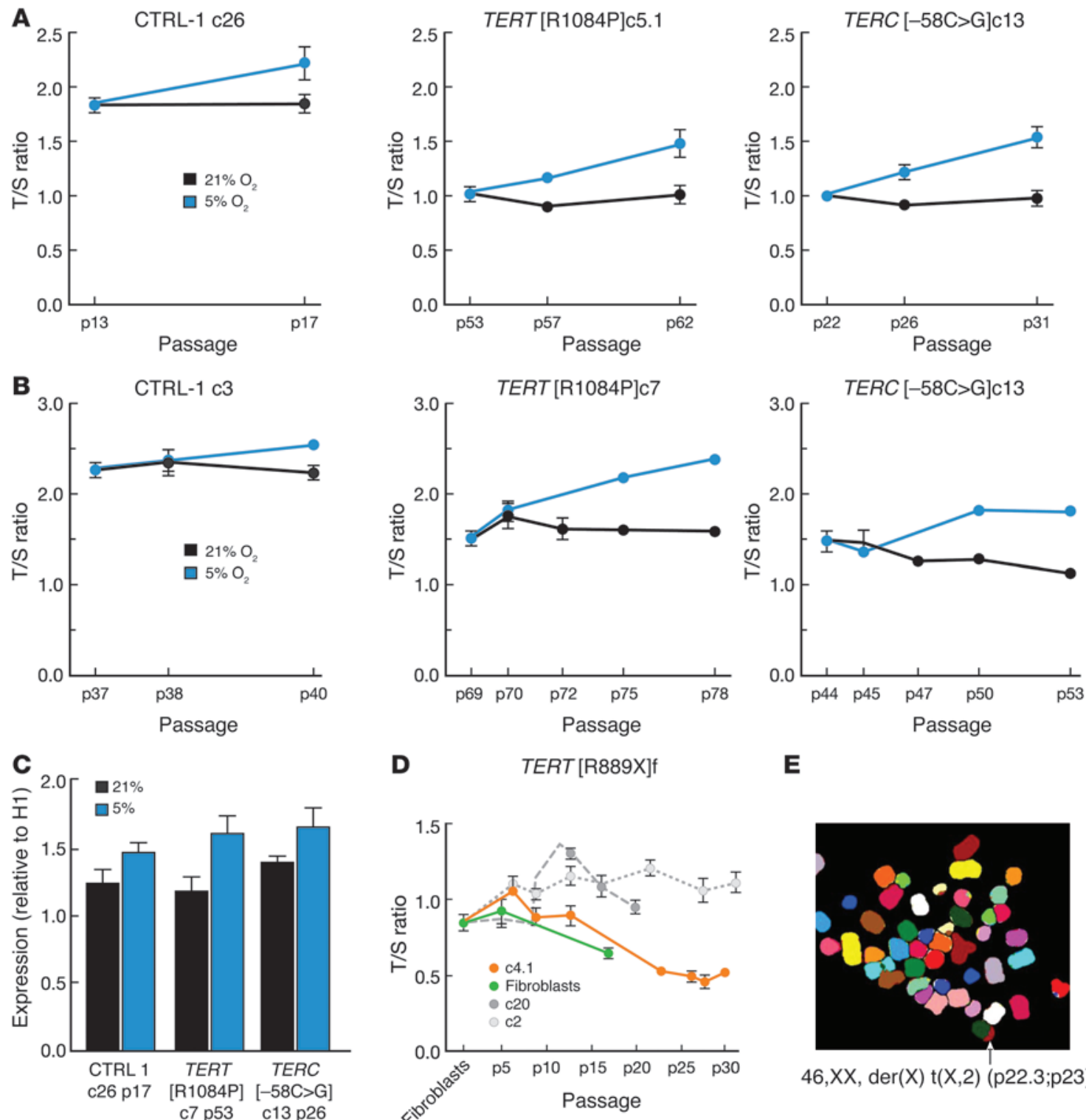

D

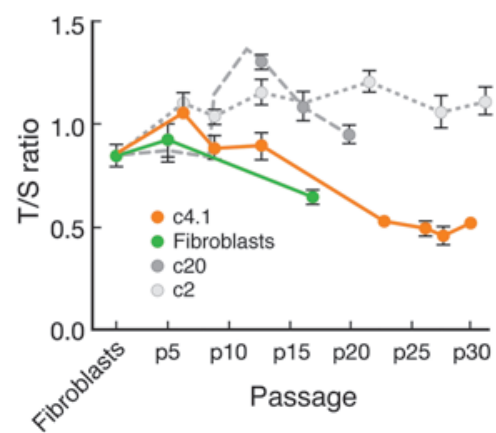

$\mathbf{E}$

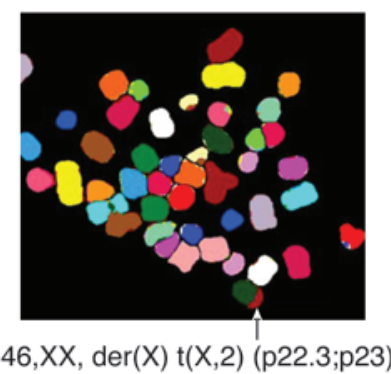

Figure 5

Environmental and genetic factors influence telomere dynamics in iPSC. (A) Telomere length over time is shown in iPSC from normal (CTRL-1 c26), TERT (TERT[R1084P] c5.1), and TERC (TERC[-58C>G]c13) mutated iPSCs cultured simultaneously in either $5 \%$ or $21 \%$ oxygen. (B) Reverse experiment: normal iPSC (CTRL-1 c3) and mutated iPSC (TERT (TERT [R1084P] c7.0, TERC (TERC[-58C>G]c13) previously cultured in $5 \%$ oxygen were either continuously cultured in $5 \%$ or placed in $21 \%$ oxygen. Telomere lengths were measured at indicated passages. (C) TERT mRNA is upregulated in iPSC cells cultured in reduced oxygen conditions (5\%). (D) Telomere attrition in clone TERT[R889X]f c4.1 (orange line) compared with other clones derived from the same starting population of patient fibroblasts. (E) Karyotype analyzed via SKY for clone TERT[R889X]f c4.1, with arrow identifying the unbalanced translocation $\operatorname{der}(\mathrm{X}) \mathrm{t}(\mathrm{X} ; 2)$.

The ability to maintain telomeres is a necessary attribute of pluripotent stem cells with unlimited replicative potential (33). The induction of telomerase expression and resultant telomere elongation occur following reprogramming of somatic cells to pluripotency in both murine (18) and human (19) iPSCs. MYC and KLF-4, 2 of the transcription factors commonly used to derive iPSCs and endogenously upregulated following reprogramming, directly or indirectly induce TERT expression $(30,34)$. Reprogramming is associated with de novo changes in methylation of subtelomeric chromosomal regions and eleva- tion of telomeric-repeat-containing RNA (TERRA) expression, indicating that significant changes in the architecture and function of telomeric and subtelomeric structures take place during this process (19).

Previous derivations of iPSCs from patients with X-linked dyskeratosis congenita and a DKC1 mutation yielded conflicting results. Agarwal et al. (20) reported significantly elongated telomeres and high TERC expression after reprogramming in single clones derived from 2 patients, but Batista et al. observed telomere erosion and low TERC expression in DKC1-mutant clones (21). The 
A
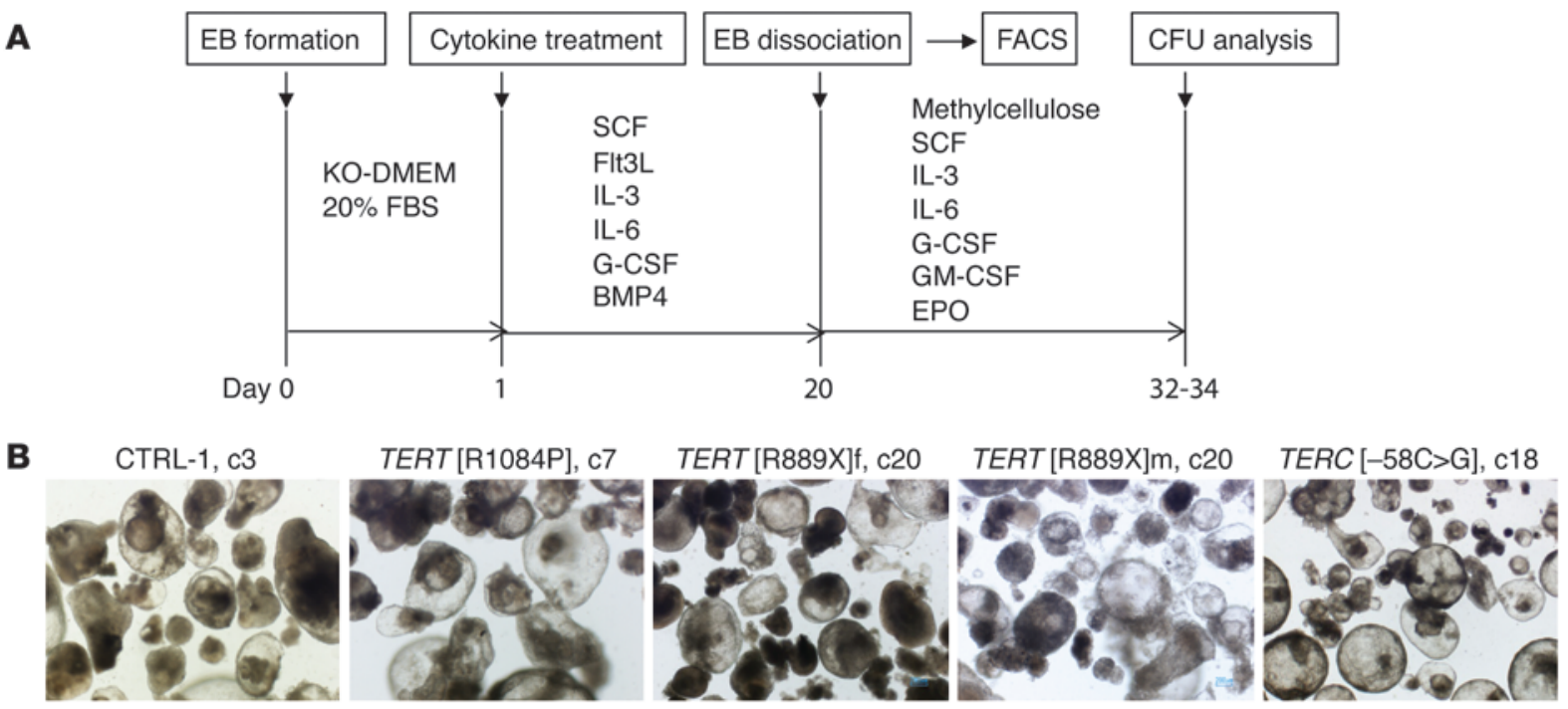

C

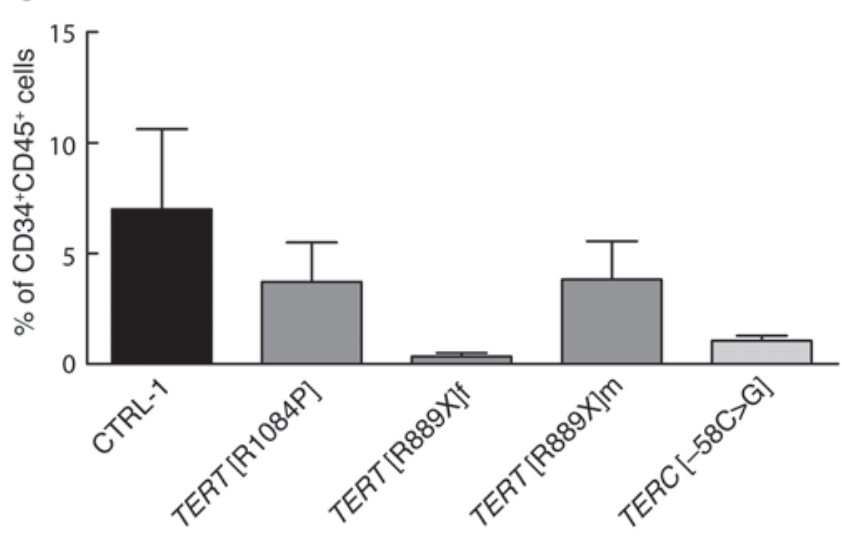

D

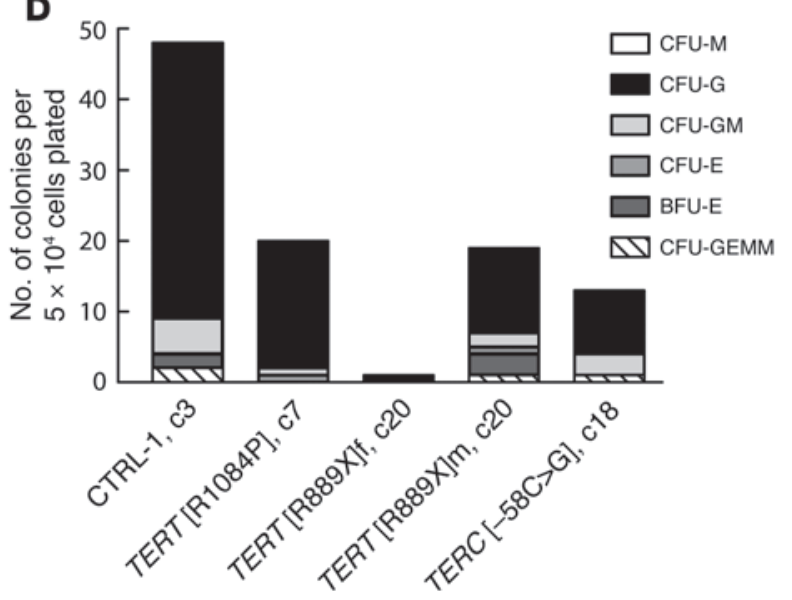

Figure 6

Hematopoietic differentiation is impaired from telomerase mutant iPSC. (A) Schematic overview of the experimental procedure. EB were cultured for 20 days in hematopoietic differentiation medium. After the dissociation of the EB, cells were plated in methylcellulose containing a cytokine cocktail that favors the differentiation of human hematopoietic progenitors toward myeloid lineages, including erythroid and megakaryocyte progenitors. (B) Similar morphologies of EB from control iPSC, and mutant iPSC on day 20 before dissociation. Original magnification, $\times 40$. (C) Flow cytometry analysis for hematopoietic differentiation markers CD34 and CD45 from 3 independent experiments. (D) Qualitative and quantitative analysis of hematopoietic colonies derived from control and mutant iPSC. CFU-GEMM, CFU-granulocyte, erythrocyte, monocyte, megakaryocyte; CFU-GM, CFU-granulocyte, macrophage; BFU-E, burst forming unit-erythroid; CFU-E, CFU-erythroid; CFU-G, CFU-granulocyte; CFU-M, CFU-macrophage.

mechanisms of telomere maintenance during reprogramming of human telomerase-mutant cells remain controversial.

We observed that TERT mRNA expression levels in TERT- and TERC-mutant iPSCs were generally increased upon reprogramming as compared with parental fibroblasts, similarly to the degree of upregulation observed during generation of control iPSCs. However TERT mRNA levels were consistently lower in iPSCs derived from 2 individuals carrying the TERT codon R889X truncation mutation. This mutation results in a premature stop codon, eliminating the enzymatic C-terminal domain from the protein. How this premature stop codon results in lower mRNA levels is not clear and may involve the mRNA nonsense-mediated decay (NMD) mechanism (35). Analysis of TERT-mutant iPSCs in the current study demonstrated that lower TERT mRNA levels might be an additional mechanism for telomerase insufficiency in some patients with truncation mutations and highlights the contribution of the reprogramming technology to identify regulatory mechanisms that would have been difficult to detect by conventional knockin and knockout models.

During early passages, the telomere elongation rate was significantly higher in control iPSCs than in TERT- or TERC-mutant iPSCs (Figure 2), and continuous elongation over the first passages indicated the observed telomere elongation in our iPSCs was not due to a selection of somatic cells with longer telomeres to begin with. These findings are consistent with results reported by Batista et al. in 2 patients with dyskeratosis congenita and heterozygous mutations in TERT (21). However, patients with dyskeratosis congenita usually have multi-organ dysfunction (skin and mucous 
membrane findings and early bone marrow failure and liver and lung manifestations) during the first decade of life, and the diagnosis requires at least 2 of 3 mucocutaneous features $(3,5,36)$; the diagnosis can be established by family history and the clinical phenotype, and the prognosis is usually poor. In contrast, patients bearing TERT or TERC mutations present with disease later in life, often only a single organ is affected, and family members with the same mutation may be clinically normal $(3,10,37)$; they have isolated aplastic anemia or pulmonary fibrosis without apparent involvement of other organs. Clinically, these patients may not be distinguishable from others with the same phenotype (aplastic anemia, pulmonary fibrosis, hepatic cirrhosis) without a telomerase mutation, and their prognosis is usually better than is that of patients with dyskeratosis congenita.

We demonstrate that iPSCs from patients with TERT or TERC mutations and aplastic anemia or marrow hypocellularity can be utilized to model the hematopoietic defect, demonstrating decreased production of hematopoietic colonies following differentiation, with the degree of the in vitro defect corresponding to the hematologic clinical status of the patient used to derive the iPSC. This successful disease modeling could be utilized for drug screening or investigation of the mechanisms linking telomere shortening to impaired hematopoietic output and may be helpful for uncovering the variable disease penetrance of telomeropathies. Differentiation of the mutant iPSC toward hepatocytes or pulmonary epithelial cells may in the future provide insights into the genesis of dysfunction in these tissues in telomeropathy patients, previously difficult to study due to lack of renewable tissue samples for experiments.

In families with TERT or TERC heterozygous mutations, clinical features may manifest earlier and with greater severity in each subsequent generation, a phenomenon termed disease anticipation (38). Our findings provide a molecular mechanism during embryogenesis for disease anticipation in telomeropathies. Although telomeres are elongated during reprogramming of telomerase-mutant somatic cells, the elongation is defective, resulting in pluripotent cells with shorter telomeres in comparison with normal, suggesting that telomere elongation during germ cell production and early embryogenesis may also be insufficient. This could effectively lead to embryos with shorter telomeres in each subsequent generation. We have recently directly demonstrated that telomere length is inherited from parents using a murine model of telomerase deficiency (39). In the offspring of animals deficient for telomerase, telomeres are shorter than in their parents. In contrast, the presence of normal telomerase expression during embryogenesis after several generations with deficient telomerase does not restore telomere length to a "normal" set point, but rather maintains telomere lengths comparable to those in their parents (40). Thus, telomerase and telomere elongation are tightly regulated during embryogenesis, and disturbances in telomerase expression may result in abnormal telomere attrition in the offspring.

Despite our demonstration of impaired telomere elongation in telomerase-mutant iPSCs, there was marked heterogeneity among individual iPSC clones derived from the same patient, between clones from different patients with identical or similar mutations, and even between individual clones measured at different passages, regarding both telomere elongation rate and directly measured telomerase activity. Of note, all cells were harvested at the same time points during culture and had a similar cell density and spontaneous differentiation proportions. These findings raise at least 3 important issues. First, iPSCs are not a stable homogeneous clonal population in culture, and culture conditions or stochastic events may result in changes in telomerase expression and function. Three different iPSC clones derived from the same starting population from a single patient carrying a TERT mutation all showed reduced telomere elongation during the first 5 passages, but 1 (TERT[R889X]f c4.1) eventually developed telomere attrition. In this clone, we identified a derivative chromosome $\operatorname{der}(X) t(X ; 2)$ in all metaphases analyzed in later passages. Apparently, acquisition of a chromosomal abnormality may change iPSC homeostasis and the ability to maintain telomeres. Genomic instability is a major concern in reprogramming, and our knowledge of how large aberrations and less obvious point mutations affect iPSC behavior is evolving (41). We also determined that oxygen concentration affects the rate of telomere elongation, as iPSCs cultured in low oxygen tension conditions consistently showed higher elongation rates and increased TERT expression regardless of telomerase complex mutation status. In agreement with our findings, Coussens et al. recently demonstrated that hypoxia-inducible factor $1 \alpha$ (HIF1- $\alpha$ ) is a regulator of TERT expression in mouse ESCs (42). In addition, oxidative damage may provoke telomere erosion in human fibroblasts (43).

Second, heterogeneity in telomere length may, at least in part, be responsible for the incongruent results of previous studies examining telomerase complex mutations on iPSC derivation and behavior. While Agarwal et al. observed telomere elongation in 1 of 2 clones derived from a DKC1-mutant patient (20), Batista et al. did not observe any elongation of telomeres in iPSCs derived from the same original DKC1-mutant fibroblasts. Instead, this study found activation of TRP53 and CDKN1A/p21 and subsequent senescence of the iPSCs (21). Agarwal et al. analyzed a very limited number of clones from only 1 mutation and protein in the telomerase complex, which may not fully reflect a heterogeneous disease or take into account the clonal heterogeneity described above, as later acknowledged by the authors (44). Our results indicate clear variability among and within clones by multiple measurements and over time in culture. However, our results following transgene excision indicate that this variability is not caused by residual expression of the reprogramming factors. A careful analysis of many clones is necessary to utilize iPSCs to study disease biology, to screen for drug activity, and to ensure that artifacts of culture are not being interpreted erroneously.

Third, heterogeneity in telomere elongation among clones may provide the molecular basis for the wide spectrum in the clinical presentation of telomere diseases, even within the same family. In the brother/sister pair (TERT[R889X]m and TERT[R889X] f) presented in our study, the clinical presentation was different; while the sister had aplastic anemia, the brother, who was older, was clinically healthy. These different phenotypes were mirrored by differences in telomere elongation in iPSCs and hematopoietic differentiation capacity between the siblings, consistent with additional genetic, epigenetic, and environmental factors that have an impact on the clinical manifestations of these diseases in germ cells and during early embryogenesis.

Finally, although telomere elongation was reduced in the reprogramming of TERT- or TERC-mutant haploinsufficient cells, it was present and resulted in iPSCs with longer telomeres than parental fibroblasts. The identification of additional cellular pathways involved may be helpful in the establishment of therapeutic strategies to boost telomerase function and telomere elongation 
in patients' hematopoietic stem cells or other tissues in vivo. For example, androgens and estrogens can induce telomerase expression in hematopoietic cells and may be of therapeutic benefit, but their effects are limited and many patients eventually fail hormone therapy (45). Our observation that hypoxia can increase telomere length, even in telomerase-mutant cells, is of interest, and investigation of the mechanisms and pathways involved may result in novel therapeutic strategies.

In conclusion, iPSCs derived from patients with telomere diseases and heterozygous telomerase mutations showed reduced and variable, but clearly present, telomere elongation. Pathways that engage telomerase activation and telomere elongation during reprogramming are potential therapeutic targets for the treatment of patients with aplastic anemia with short telomeres. iPSCs could indeed serve as a valuable tool to test these modalities in vitro, but the variability of this model should be considered carefully.

\section{Methods}

Derivation of skin fibroblast cells from patient and healthy volunteer. Patients were selected according to the following requirements: (a) heterozygous mutation in either TERT or TERC, (b) decreased telomere lengths compared with age-matched controls, and (c) a family history of telomere disease (bone marrow failure, lung disease, or liver disease). The healthy male control-1 had normal telomere length and no family history of these diseases. Dermal fibroblasts were obtained by punch biopsy from the upper medial arm. Biopsies were sectioned into approximately $1 \mathrm{~mm}^{3}$ pieces and placed in $10 \mathrm{ml}$ PBS containing $100 \mathrm{mg} / \mathrm{ml}$ collagenase II, $2.5 \mathrm{U} / \mathrm{ml}$ dispase, and $10 \mathrm{U} / \mu \mathrm{l}$ DNaseI (all Gibco; Invitrogen). Skin fragments were incubated for 50 minutes on an orbital shaker $(200 \mathrm{rpm})$ at $37^{\circ} \mathrm{C}$. Following a washing step with complete culture medium (DMEM; Gibco, Invitrogen), 10\% FBS (Sigma-Aldrich), and 1\% penicillin-streptomycin-glutamine (PSG) (Gibco, Invitrogen), the skin pieces were placed in a 6-well tissue culture plate, covered with a glass cover slip, and cultured for approximately 7-10 days in complete culture medium. After initial fibroblast-like cells migrated from the tissue, the cover slip was taken off and the cells were enzymatically (Trypsin $0.05 \%$; Gibco, Invitrogen) passaged to a fresh tissue culture dish. Following the initial derivation procedure, all the cells were passaged according to standardized procedure, with a starting cell number of 10,000 cells $/ \mathrm{cm}^{2}$.

Generation of iPSCs and culture of pluripotent cells. iPSCs were generated from human dermal fibroblasts by forced expression of the reprogramming factors OCT4, SOX2, KLF4, and MYC using either retroviral vectors expressing the 4 factors individually (pMIG-OCT4, pMIG-SOX2, and pMIG-KLF4, Addgene; pMIG containing MYC was provided by George Q. Daley, Children's Hospital, Boston, Massachusetts, USA) or all combined form the polycistronic lentiviral vector STEMCCA provided by G. Mostoslavsky (Boston University, Boston, Massachusetts, USA) (27). The production of the viral particles was done as described $(26,27)$. Five days following transduction, fibroblasts were transferred to plates coated with mouse embryonic fibroblasts (MEFs) and grown in ES cell medium (ES medium, DMEM/F12, supplemented with $20 \%$ knockout serum replacement [KSR], $0.1 \mathrm{mM}$ nonessential amino acids, $1 \mathrm{mM}$ L-glutamine [all Gibco, Invitrogen], $10 \mathrm{ng} / \mathrm{ml}$ recombinant human fibroblast like growth factorbasic [Peprotech], and $0.1 \mathrm{mM} 2$-mercaptoethanol [Sigma-Aldrich]). From day 5 to day 12, $0.5 \mathrm{mM}$ valproic acid (Stemgent) was added to enhance efficiency of iPSC generation in some cultures (ref. 46 and Supplemental Table 1). Between days 14 and 30, individual, GFP-negative (for PMIG vectors) ES cell-like colonies were picked and expanded on MEFs. iPSC clones were enzymatically passaged using collagenase IV (Gibco, Invitrogen). In order to avoid any influence of MEFs used in the coculture system on further analyses, iPSCs were harvested and dispersed to single cells using trypsin $(0.05 \%)$ and purified away from residual MEFs via negative selection using MACS separation with antibodies directed against a murine fibroblast surface protein (Miltenyi). iPSCs were cultured in 21\% oxygen tension unless otherwise designated.

In order to rule out the potential influence of the reprogramming transgenes on telomere behavior, the transgene cassette was excised using the cre-recombinase-expressing vector pCL20i4rEF1-Puro-T2A-cre-GFP provided by Harry L. Malech (National Institute of Allergy and Infectious Diseases, Bethesda, Maryland, USA). Briefly, iPSC were plated on Matrigel (BD), cultured in MEF-conditioned ES medium, and transfected with pCL20i4rEF1-Puro-T2A-cre-GFP the next day using Lipofectamine LTX \& Plus Reagent (Invitrogen) according to the manufacturer's recommendations. Transduced cells were positively selected for 3 days in Puromycine (day $1: 3 \mu \mathrm{g} / \mathrm{ml}$, day 2 and day $3: 2 \mu \mathrm{g} / \mathrm{ml}$, Sigma-Aldrich) containing conditioned ES medium. Approximately 10 days after the transfection, iPSC colonies were picked, expanded, and analyzed by Southern blot for excision of transgenes. Excised iPSC subclones stained positive for common pluripotency markers (Supplemental Table 3, data not shown) and were maintained in culture for at least 10 passages after the excision.

Immunohistochemistry for pluripotency markers. Cells were cultured with MEFs in 96-well plates for 3-5 days to allow for several colonies to establish growth. Cells were washed 1 time with PBS and fixed with $100 \mu \mathrm{l} 4 \%$ paraformaldehyde (Sigma-Aldrich) for 30 minutes at room temperature. For intracellular staining, cells were permeabilized with $100 \mu 10.2 \%$ Triton X-100 (Sigma-Aldrich) for 30 minutes at room temperature followed by an additional washing step with PBS and then incubated for 2 hours at room temperature in $100 \mu \mathrm{l}$ blocking buffer (3\% BSA $/ 5 \%$ donkey serum; Jackson ImmunoResearch Laboratories). After blocking, cells were incubated with $100 \mu \mathrm{l}$ primary antibody $\left(1: 100\right.$ in blocking buffer) overnight at $4{ }^{\circ} \mathrm{C}$. When necessary, cells were incubated with $100 \mu \mathrm{l}$ secondary antibody (1:500 in blocking buffer) for 3 hours at $4^{\circ} \mathrm{C}$. Following incubation with antibodies, cells were washed with PBS, and nuclei were stained using Vectashield with DAPI (Vector Laboratories). An overview of the antibodies used can be found in Supplemental Table 3.

In vivo differentiation assay (teratoma formation). All mice were bred and maintained at the NIH animal facility. Immune-deficient NOD.CgPrkdcScid Il2rgtm1wjl/SzJ (NSG) male and female mice 6 to 16 weeks of age were used as recipients for teratoma assays. Subcutaneous (nuchal region) and intramuscular injections (left thigh) were employed. iPSCs were harvested using collagenase IV as described above, spun down at $200 \mathrm{~g}$ for 3 minutes, and resuspended in $115 \mu \mathrm{l}$ (subcutaneous injection) or $65 \mu \mathrm{l}$ (intramuscular injection) of cold Iscove's Modified Dulbecco's Media (IMDM; Gibco, Invitrogen) and complemented with the same volume of cold Matrigel (BD). Approximately $2.0 \times 10^{6}$ cells were injected per site. Following development of visible tumors between 7 and 12 weeks, mice were euthanized and tumors were removed, fixed in Bouin's solution (Sigma-Aldrich), and processed (cutting and H\&E staining) by the NHLBI pathology core facility.

Hematopoietic differentiation assay. Hematopoietic differentiation of iPSCs was performed according to a previously described procedure (47). Briefly, undifferentiated iPSCs were harvested using collagenase type IV and incubated in ultra-low attachment plate (Corning) with EB formation medium KnockOut DMEM (Gibco, Invitrogen) supplemented with $20 \%$ nonheat-inactivated FBS (Atlanta Biologicals), $0.5 \mathrm{mM}$ L-glutamine, $0.1 \mathrm{mM}$ nonessential amino acids, and $0.1 \mathrm{mM} 2$-mercaptoethanol. After 24 hours, medium was replaced with hematopoietic differentiation medium (EB formation medium supplemented with $10 \mathrm{ng} / \mathrm{ml} \mathrm{IL-3,} 10 \mathrm{ng} / \mathrm{ml} \mathrm{IL-6,}$ $25 \mathrm{ng} / \mathrm{ml}$ bone morphogenetic protein 4 [all R\&D Systems], $300 \mathrm{ng} / \mathrm{ml}$ flt3 ligand [Flt3L] [Miltenyi], $50 \mathrm{ng} / \mathrm{ml}$ granulocyte CSF [G-CSF] [filgras- 
tim; Amgen], and $300 \mathrm{ng} / \mathrm{ml} \mathrm{SCF}$ [Amgen]). Medium was replaced with fresh hematopoietic differentiation medium every $4-5$ days. On day 20 , EBs were dissociated into single cells and analyzed by flow cytometry or plated in CFU assays. For flow cytometry, cells were stained for viability using LIVE/DEAD Fixable Violet Dead Cell Stain Kit (Invitrogen) and CD34 and CD45 (BD). Data were acquired on a BD LSRII flow cytometer (BD) and analyzed using FlowJo software (TreeStar). For CFU assays, 50,000 EB-derived cells were plated in MethoCult H4435 medium containing SCF, IL-3, IL-6, G-CSF, GM-CSF, and erythropoietin (EPO) (Stem Cell Technologies) for 12-14 days.

Mutation analysis. Total genomic DNA was extracted from cell pellets using the automated Maxwell 16 LEV Blood DNA Purification Kit and quantified by Nanodrop. PCR amplification of TERT and TERC genes was performed as previously described, and PCR products were purified with the QIAquick PCR Purification Kit (QIAGEN) (6). Direct sequencing was performed with BigDye Terminator version 3.1 and products analyzed in an automated genetic sequence analyzer (ABI Prism 3100; Applied Biosystems). The list of primers used for this analysis can be found in Supplemental Table 2.

SKY. SKY analysis was performed as previously described (28). All aberrations described were in accordance with the International System for Human Cytogenetic Nomenclature (48). Clonal aberrations were defined as the presence of a minimum of at least 2 cells sharing the same chromosomal gain or structural alteration. A loss of chromosome was defined as clonal if this abnormality was detected in at least 3 cells.

Southern hybridization for telomere length. Southern blot analysis was performed using $275 \mathrm{ng}$ of genomic DNA for each sample, following digestion with Hinf1 and Rsa1. Using the TeloTAGGG Telomere Length Assay kit (Roche), DNA digestion, Southern analysis, and determination of mean terminal restriction fragment (TRF) length were performed according to the manufacturer's protocols.

Southern blot analysis to detect the transgenes expressed from the STEMCCA provirus were performed as previously described (49). Briefly, $10 \mu \mathrm{g}$ of genomic DNA of each sample was digested with BamHI (Fermentas). After blotting, a 541-bp, p32-labeled DNA fragment complementary to the proviral wpr-element was used to detect the nonexcised, integrated proviral DNA. The primer information to generate the probe can be found in Supplemental Table 2.

qPCR for telomere length. qPCR was performed as described by Cawthon $(29,50)$, with several modifications. PCR reactions were pippetted in triplicate using the Qiagility robot (QIAGEN) and consisted of SYBR Green PCR Master Mix (QIAGEN), forward and reverse primers, and $1.6 \mathrm{ng}$ of DNA per reaction. Amplification and quantification using the Rotor-Gene $\mathrm{Q}$ (QIAGEN) was performed twice: first using primers for telomeric repeats (T) and second using primers for the single-copy gene $36 B 4(\mathrm{~S})$. Primer sequences can be found in Supplemental Table 2. The average telomere length for each sample was calculated as a relative $\mathrm{T} / \mathrm{S}$ ratio and normalized to a standard control DNA (average telomere length by Southern blot, $\left.8.6 \mathrm{~kb} ; 2^{\Delta \Delta \mathrm{Ct}}\right)$. The final result for a given sample represents the mean and SEM for at least 3 independent assays. In each run, a standard curve was generated using the standard control DNA at various quantities (10, 5, $2.5,1.25$, and $0.625 \mathrm{ng} /$ reaction). Two validation samples were run twice in each plate (the standard control DNA and an umbilical cord blood sample), and data were only accepted if the results for the 2 validation samples were within $5 \%$ of expected values. Based on control and validation runs, the intra-assay variation of the method was $1.2 \%$ coefficient of variation and the inter-assay variation was $3.1 \%$ for the validation samples. Samples for each clone at different passages were run in the same experiment and in at least 2 independent runs. In control experiments, the correlation between Southern blot and qPCR was $r^{2}=0.86$.
Real-time RT-PCR. RT-PCR was performed using the Rotor-Gene Multiplex RT-PCR kit (QIAGEN) according to the manufacturer's recommendations. Briefly, cell lysates were homogenized using the QIAshredder Kit (QIAGEN) and total RNA was extracted using the RNeasy Mini Kit (QIAGEN). RNA was quantified by Nanodrop analysis. Multiplex reactions consisted of the kit-supplied master mix and reverse transcriptase as well as primer-probe mixes for TERT (Fam), TERC (Tye), and ACTIN (MAX) (each primer, $0.4 \mu \mathrm{M}$; each probe, $0.2 \mu \mathrm{M}$ ), and $50 \mathrm{ng}$ of RNA. Standard curves were generated using serial dilutions of TERT, TERC, and actin plasmids. Primer sequence information can be found in Supplemental Table 2. Each reaction was performed in triplicate using a Rotor-Gene Q 5plex Platform (QIAGEN). The final result given for a sample represents the mean and SEM.

Telomeric repeat amplification protocol for telomerase activity. In vitro telomerase activity was assessed using the TRAPeze XL Telomerase Detection Kit (Millipore) according to the manufacturer's instructions. Protein from cell pellets was extracted using CHAPS lysis buffer. Reactions consisted of $300 \mathrm{ng}$ of extracted protein, telomeric substrate, fluorescent primers, dNTPs, and Taq polymerase. The mixture was incubated at $30^{\circ} \mathrm{C}$ for 30 minutes to allow for telomerase-mediated elongation of telomeric repeats and then subjected to Taq polymerase-mediated PCR amplification of the telomeric products generated. Quantification was achieved using fluorescence spectroscopy. Each reaction was performed in triplicate and normalized to the activity of an equivalent amount of HeLa protein extract. Each experiment included a positive (HeLa protein extract) and 3 negative controls (no template, no Taq-polymerase, and parental fibroblast protein extract). The final result given for a sample represents the mean and SEM.

Expression array. ST-cDNA from samples was fragmented, biotin labeled, and hybridized on Affymetrix Human Exon 1.0 ST microarray according to the manufacturer's protocol (Affymetrix). The raw data were preprocessed using the gene-level extended RMA-sketch method by Affymetrix Expression Console (EC) Software (Affymetrix). The annotation of 133,672 extended transcript clusters was based on the current version annotation file from Affymetrix (HuEx-1_0-st-v2.na32.hg19.transcript.csv). Hierarchical cluster analysis was performed by the similarity matrix (squared Pearson's correlation coefficient) on normalized intensities on extended gene level summarization crossing all the samples.

C-circle assay. We performed the C-circle assay developed by Jeremy Henson and Roger Reddel as described by the authors (51), except that DIGlabeled telomeric probes were used (Roche).

Statistics. Prism 5 software (GraphPad) was used in statistical analyses and graph creation. ANOVA and Student's $t$ test (2 tailed) were used in comparing rates of elongation. Nonparametric statistics (Kruskal-Wallis and Dunn's post test) were used in comparing grouped samples for telomerase expression and activity. A 2-tailed Student's $t$ test was used to analyze the TERT and TERC expression of excised and nonexcised iPSC clones. $P<0.05$ was considered statistically significant. Data are presented as mean \pm SEM in all figure panels in which error bars are shown.

Study approval. All human subject material was collected at the NIH Clinical Center under approval of the NHLBI institutional review board (04-H0012 and 07-H-0113), following written informed consent. All mice were enrolled in protocols approved by the NIH Heart Lung and Blood Institute Animal Care and Use Committee (H-0084R2).

\section{Acknowledgments}

This research was supported by the Divisions of Intramural Research at the NHLBI, the National Human Genome Research Institute, the National Cancer Institute, and the National Center for Regenerative Medicine at the NIH. R.T. Calado was supported 
by a FAPESP (grant 98/14247-6). The authors have no conflicts of interest to declare. We would like to thank Jichun Chen, Marie Desierto, and $\mathrm{Zu} \mathrm{Xi}$ Yu from the NHLBI pathology core facility for their support during the teratoma assays. We thank Dara Wangsa for providing the probes for SKY analysis and Vicky Guo for assisting with the iPSC culture. We are also grateful to Sachiko Kajigaya for her excellent technical support.

1. de Lange T. How telomeres solve the end-protection problem. Science. 2009;326(5955):948-952.

2. Venteicher AS, et al. A human telomerase holoenzyme protein required for Cajal body localization and telomere synthesis. Science. 2009 ; 323(5914):644-648.

3. Calado RT, Young NS. Telomere diseases. NEnglJ Med. 2009;361(24):2353-2365.

4. Kirwan M, Dokal I. Dyskeratosis congenita, stem cells and telomeres. Biochim Biophys Acta. 2009; 1792(4):371-379

5. Savage SA, Alter BP. Dyskeratosis congenita. Hematol Oncol Clin North Am. 2009;23(2):215-231.

6. Yamaguchi $\mathrm{H}$, et al. Mutations in TERT, the gene for telomerase reverse transcriptase, in aplastic anemia. NEngl J Med. 2005;352(14):1413-1424.

7. Armanios MY, et al. Telomerase mutations in families with idiopathic pulmonary fibrosis. $N$ Engl J Med. 2007;356(13):1317-1326.

8. Tsakiri KD, et al. Adult-onset pulmonary fibrosis caused by mutations in telomerase. Proc Natl Acad Sci US A. 2007;104(18):7552-7557.

9. Calado RT, et al. Constitutional telomerase mutations are genetic risk factors for cirrhosis. Hepatology. 2011;53(5):1600-1607.

10. Calado RT, et al. A spectrum of severe familial liver disorders associate with telomerase mutations. PLoS One. 2009;4(11):e7926.

11. Hartmann D, et al. Telomerase gene mutations are associated with cirrhosis formation. Hepatology. 2011;53(5):1608-1617.

12. Calado RT, et al. Constitutional hypomorphic telomerase mutations in patients with acute myeloid leukemia. Proc Natl Acad Sci U S A. 2009; 106(4):1187-1192.

13. Kirwan M, et al. Dyskeratosis congenita and the DNA damage response. Br J Haematol. 2011; 153(5):634-643.

14. Takahashi K, Yamanaka S. Induction of pluripotent stem cells from mouse embryonic and adult fibroblast cultures by defined factors. Cell. 2006;126(4):663-676

15. Thomson JA, et al. Embryonic stem cell lines derived from human blastocysts. Science. 1998; 282(5391):1145-1147.

16. Takahashi $\mathrm{K}$, et al. Induction of pluripotent stem cells from adult human fibroblasts by defined factors. Cell. 2007;131(5):861-872.

17. Maherali N, et al. Directly reprogrammed fibroblasts show global epigenetic remodeling and widespread tissue contribution. Cell Stem Cell. 2007; 1(1):55-70.

18. Marion RM, et al. Telomeres acquire embryonic stem cell characteristics in induced pluripotent stem cells. Cell Stem Cell. 2009;4(2):141-154.

19. Yehezkel S, et al. Reprogramming of telomeric regions during the generation of human induced
Received for publication October 4, 2012, and accepted in revised form February 14, 2013.

Address correspondence to: Cynthia E. Dunbar, Hematology Branch, NHLBI/NIH, 10 Center Drive, Bldg. 10/CRC, Rm. 4E-5132, Bethesda, Maryland 20892-1202, USA. Phone: 301.402.1363; Fax: 301.496.8396; E-mail: dunbarc@nhlbi.nih.gov. pluripotent stem cells and subsequent differentiation into fibroblast-like derivatives. Epigenetics. 2011;6(1):63-75

20. Agarwal S, et al. Telomere elongation in induced pluripotent stem cells from dyskeratosis congenita patients. Nature. 2010;464(7286):292-296.

21. Batista LF, et al. Telomere shortening and loss of self-renewal in dyskeratosis congenita induced pluripotent stem cells. Nature. 2011; 474(7351):399-402.

22. Ezashi T, Das P, Roberts RM. Low O2 tensions and the prevention of differentiation of hES cells. Proc Natl Acad Sci U S A. 2005;102(13):4783-4788.

23. Yoshida Y, Takahashi K, Okita K, Ichisaka T, Yamanaka S. Hypoxia enhances the generation of induced pluripotent stem cells. Cell Stem Cell. 2009; 5(3):237-241.

24. El-Chemaly S, et al. Natural history of pulmonary fibrosis in two subjects with the same telomerase mutation. Chest. 2011;139(5):1203-1209.

25. Aalbers AM, Kajigaya S, van den Heuvel-Eibrink MM, van der Velden VH, Calado RT, Young NS. Human telomere disease due to disruption of the CCAAT box of the TERC promoter. Blood. 2012;119(13):3060-3063.

26. Park IH, Lerou PH, Zhao R, Huo H, Daley GQ. Generation of human-induced pluripotent stem cells. Nat Protoc. 2008;3(7):1180-1186.

27. Sommer CA, Stadtfeld M, Murphy GJ, Hochedlinger K, Kotton DN, Mostoslavsky G. Induced pluripotent stem cell generation using a single lentiviral stem cell cassette. Stem Cells. 2009; 27(3):543-549.

28. Schrock E, et al. Multicolor spectral karyotyping of human chromosomes. Science. 1996; 273(5274):494-497.

29. Cawthon RM. Telomere measurement by quantitative PCR. Nucleic Acids Res. 2002;30(10):e47.

30. Hoffmeyer $\mathrm{K}$, et al. Wnt/beta-catenin signaling regulates telomerase in stem cells and cancer cells. Science. 2012;336(6088):1549-1554.

31. Cesare AJ, Reddel RR. Alternative lengthening of telomeres: models, mechanisms and implications. Nat Rev Genet. 2010;11(5):319-330.

32. Henson JD, et al. DNA C-circles are specific and quantifiable markers of alternative-lengthening-of-telomeres activity. Nat Biotechnol. 2009; 27(12):1181-1185.

33. Allen ND, Baird DM. Telomere length maintenance in stem cell populations. Biochim Biophys Acta. 2009; 1792(4):324-328

34. Wu KJ, et al. Direct activation of TERT transcription by c-MYC. Nat Genet. 1999;21(2):220-224.

35. Chang YF, Imam JS, Wilkinson MF. The nonsensemediated decay RNA surveillance pathway. Annu Rev Biochem. 2007;76:51-74

36. Dokal I. Dyskeratosis congenita. A disease of pre- mature ageing. Lancet. 2001;358:S27.

37. Diaz de Leon A, et al. Telomere lengths, pulmonary fibrosis and telomerase (TERT) mutations. PLoS One. 2010;5(5):e10680.

38. Vulliamy T, Marrone A, Szydlo R, Walne A, Mason PJ, Dokal I. Disease anticipation is associated with progressive telomere shortening in families with dyskeratosis congenita due to mutations in TERC. Nat Genet. 2004;36(5):447-449.

39. Chiang YJ, Calado RT, Hathcock KS, Lansdorp PM, Young NS, Hodes RJ. Telomere length is inherited with resetting of the telomere set-point. Proc Natl Acad SciU S A. 2010;107(22):10148-10153.

40. Armanios M, Alder JK, Parry EM, Karim B, Strong MA, Greider CW. Short telomeres are sufficient to cause the degenerative defects associated with aging. Am J Hum Genet. 2009;85(6):823-832.

41. Martins-Taylor K, Xu RH. Concise review: Genomic stability of human induced pluripotent stem cells. Stem Cells. 2012;30(1):22-27.

42. Coussens $\mathrm{M}$, et al. RNAi screen for telomerase reverse transcriptase transcriptional regulators identifies HIF1alpha as critical for telomerase function in murine embryonic stem cells. Proc Natl Acad Sci U S A. 2010;107(31):13842-13847.

43. von Zglinicki T, Pilger R, Sitte N. Accumulation of single-strand breaks is the major cause of telomere shortening in human fibroblasts. Free Radic Biol Med. 2000;28(1):64-74.

44. Agarwal S, Daley GQ. Telomere dynamics in dyskeratosis congenita: the long and the short of iPS. Cell Res. 2011;21(8):1157-1160.

45. Calado RT, et al. Sex hormones, acting on the TERT gene, increase telomerase activity in human primary hematopoietic cells. Blood. 2009;114(11):2236-2243.

46. Huangfu D, et al. Induction of pluripotent stem cells by defined factors is greatly improved by small-molecule compounds. Nat Biotechnol. 2008; 26(7):795-797.

47. Chadwick K, et al. Cytokines and BMP-4 promote hematopoietic differentiation of human embryonic stem cells. Blood. 2003;102(3):906-915.

48. Scaffer LG, Slovak ML, Campbell LJ, eds. An International System for Human Cytogenetic Nomenclature. Basel, Switzerland: S. Karger Publishing; 2009.

49. Sommer CA, et al. Excision of reprogramming transgenes improves the differentiation potential of iPS cells generated with a single excisable vector. Stem Cells. 2010;28(1):64-74.

50. Cawthon RM. Telomere length measurement by a novel monochrome multiplex quantitative PCR method. Nucleic Acids Res. 2009;37(3):e21.

51. Henson JD, et al. A robust assay for alternative lengthening of telomeres in tumors shows the significance of alternative lengthening of telomeres in sarcomas and astrocytomas. Clin Cancer Res. 2005; 11(1):217-225. 